\title{
Un continent, deux blocs, trois idees. Le COMECON, la CEE et le processus d'Helsinki
}

\author{
Nicolas BADALASSI
}

\begin{abstract}
Au tournant des années 1960 et 1970, l'URSS entend se servir de la Conférence sur la Sécurité et la Coopération en Europe pour accroître ses relations économiques et commerciales avec les pays de la CEE tout en luttant contre la construction européenne à l'Ouest. Elle promeut l'idée d'intégration est-européenne pour parvenir à terme à l'unification économique paneuropéenne. Elle tente ainsi de faire reconnaître le Comecon comme étant l'équivalent socialiste du Marché commun, d'obtenir l'octroi de la clause de la nation la plus favorisée et de mettre sur pied un organisme de sécurité paneuropéen. Mais, à chaque fois, elle doit faire face à l'opposition des pays de la CEE.
\end{abstract}

Keywords: COMECON, CEE, CSCE, paneuropéanisme, guerre froide

Le processus d'Helsinki est aujourd'hui considéré par les historiens des relations internationales comme l'un des facteurs ayant conduit à la fin de la guerre froide. Les dispositions de la Conférence sur la Sécurité et la Coopération en Europe en matière d'échanges culturels et de contacts humains ont en effet permis aux dissidents du bloc de l'Est, dès la deuxième moitié des années 1970, de disposer d'un document multilatéral sur lequel s'appuyer pour faire entendre leurs revendications. Pourtant, à l'origine, le projet soviétique de conférence avait un tout autre but, que les Occidentaux ont réussi à retourner en leur faveur.

Au milieu des années 1960, alors que la détente s'installe progressivement dans les relations Est-Ouest, l'URSS de Brejnev confère à sa politique européenne un objectif central : il s'agit de faire reconnaître aux Occidentaux la mainmise soviétique sur l'Europe orientale et, plus généralement, le statu quo politique et territorial européen. Moscou veut ainsi geler la division Est-Ouest afin de pouvoir faire face à la menace grandissante de la Chine qui ose remettre en question la suprématie soviétique au sein du mouvement communiste international.

Pour parvenir à ses fins, le Kremlin promeut, auprès des pays d'Europe occidentale, l'idée de réunir une conférence paneuropéenne de sécurité qui, rassemblant l'ensemble des Etats du continent, pourrait consacrer l'existence des deux blocs. L'emploi du qualificatif « paneuropéen » montre néanmoins que Brejnev est disposé à aller plus loin que le simple statu quo : une telle conférence pourrait aussi contribuer à émousser la solidarité occidentale. En effet, lorsque les Soviétiques émettent leur proposition, en 1965, les rapports entre pays de l'Ouest n'ont jamais été aussi tendus depuis le début de la guerre froide, ce pour plusieurs raisons : la guerre que mènent les Etats-Unis au Vietnam est très impopulaire en Europe occidentale et nuit aux relations transatlantiques, la «crise de la chaise vide » 
due à l'intransigeance du général de Gaulle freine la construction européenne, la politique pro-américaine du chancelier ouest-allemand Ludwig Erhard a mis entre parenthèses le partenariat franco-allemand voulu par le président français. Dans ce contexte, l'idée de conférence paneuropéenne apparaît comme un moyen, pour les Soviétiques, de tirer profit des tensions intra-occidentales. Ils développent ainsi un véritable projet de sécurité collective : la détente rendant caducs l'OTAN et le pacte de Varsovie, on pourrait remplacer ces deux alliances militaires par un organisme paneuropéen de sécurité qui couvrirait tout l'espace s'étendant de l'Atlantique à l'Oural. Lorsqu'en 1969-1970, les dirigeants occidentaux finissent par donner leur accord à l'organisation d'une Conférence sur la Sécurité et la Coopération en Europe en échange de l'insertion dans son ordre du jour des thèmes des droits de l'homme et de la circulation des hommes, des idées et des informations, aux objectifs initiaux de l'URSS s'est ajouté un nouvel impératif, de taille : il est indispensable d'accroître les relations économiques et commerciales avec l'Occident, le PIB des pays du bloc socialiste étant en perpétuelle diminution à partir du milieu des années 1960. La nécessité de se rapprocher du Marché commun pour accroître les échanges économiques avec l'Ouest, conjuguée aux objectifs structurels que Moscou confère à la CSCE, conduit les Soviétiques à élaborer une stratégie visant à répondre à ces impératifs mais sans les opposer les uns aux autres. Le Kremlin cherche, via la conférence, à réaliser un double processus d'intégration : est-européen d'abord, paneuropéen ensuite. Il s'agit de créer un partenariat privilégié entre la CEE et le Comecon en faisant reconnaître aux Occidentaux que le second est l'équivalent socialiste de la première. Moscou entend pour ce faire renforcer l'intégration au sein du Comecon pour obtenir, à terme, la désintégration de la CEE et l'instauration d'un cadre paneuropéen de coopération s'étendant de l'Atlantique à l'Oural. A la CSCE, le désir de parvenir à la réalisation de ce double objectif se manifeste lors des débats dévolus aux suites de la conférence et au moment des discussions relatives à la « deuxième corbeille d'Helsinki » ${ }^{1}$ sur la coopération économique et commerciale Est-Ouest. Celle-ci est l'objet, à Genève, d'âpres négociations qui opposent les pays du pacte de Varsovie à ceux de la Communauté européenne.

Cette contribution propose d'analyser les arguments avancés par les pays socialistes pour justifier leurs projets d'intégration est-européenne et paneuropéenne. Il s'agit également de voir comment ces plans sont systématiquement contrecarrés par les Etats de la Communauté européenne.

1 Les thèmes discutés à la CSCE sont répartis en trois « corbeilles ». La première traite des questions de sécurité européenne proprement dite ; la deuxième regroupe les questions économiques, scientifiques et techniques ; la troisième a trait à la circulation des hommes, des idées et des informations. Deux autres thèmes figurent à l'ordre du jour de la CSCE mais demeurent hors corbeilles : la sécurité et la coopération en Méditerranée et les suites à donner au processus d'Helsinki. 
$\mathrm{Si}$, malgré tous leurs efforts, les Soviétiques échouent à établir un partenariat entre le Comecon et la CEE (I), ils tentent de compenser cet échec en obtenant des pays du Marché commun qu'ils accordent à tous les Etats de la CSCE la clause de la nation la plus favorisée (II) et en insistant pour mettre sur pied un organisme paneuropéen permanent destiné à leur conférer un droit de regard sur les affaires ouest-européennes (III).

\section{CEE et Comecon, l'impossible partenariat}

Dans le discours qu'il prononce lors de la première phase de la CSCE en juillet $1973^{2}$, le ministre soviétique des Affaires étrangères Andreï Gromyko préconise l'organisation en Europe d'une coopération économique à long terme, tant multilatérale que bilatérale : cette affirmation laisse penser aux pays de l'OTAN que l'un des buts essentiels de l'URSS à la CSCE est d'obtenir un plus large accès aux ressources financières, à la science et à la technologie de l'Occident, accès qu'elle n'a pu s'assurer bilatéralement ou par des négociations avec la $\mathrm{CEE}^{3}$.

En outre, le Kremlin cherche de nouveaux moyens pour remédier à la diminution continue du taux de croissance économique de l'URSS. De fait, Brejnev et Gromyko savent qu'il est temps pour eux d'admettre la viabilité de la CEE pour en tirer profit ${ }^{4}$. Jusqu'alors, celle-ci était perçue comme un bloc commercial fermé et discriminatoire, une simple émanation ouest-européenne de l'OTAN accusée de maintenir la division économique du continent.

L'URSS infléchit sa position en janvier 1972 et semble la modifier en mars lorsque Brejnev évoque devant le Congrès soviétique des unions du commerce les possibilités de coopération entre le Marché commun et les pays socialistes, reconnaissant de fait que la CEE est une réalité. L'initiative est suivie, en décembre 1972, par une proposition du Secrétaire général du PCUS d'ouvrir des négociations sur un accord commercial entre la CEE et le Comecon ${ }^{5}$. A l'Ouest, on perçoit

2 Tandis que les pourparlers préparatoires multilatéraux (PMP) de la CSCE durent de novembre 1972 à juin 1973, la conférence proprement dite est constituée de trois phases : la première, du 3 au 7 juillet 1973, permet aux trente-cinq ministres des Affaires étrangères rassemblés à Helsinki de valider l'ordre du jour fixé durant les PMP ; la deuxième, qui se déroule à Genève, correspond à la phase de négociation du contenu de l'Acte final et s'étale entre septembre 1973 et juillet 1975 ; la troisième réunit à Helsinki du 30 juillet au $1^{\text {er }}$ août 1975 les chefs d'Etat et de gouvernement des trente-cinq pays représentés en vue de la signature de l'Acte final.

3 Document OTAN, C-M (73) 75, 17 septembre 1973. Archives du Quai d'Orsay (AMAE), CSCE, vol. 29.

4 Marie-Pierre Rey, "L'URSS et l'Europe communautaire, représentations et pratiques, 19571991", in Anne Deighton, Gérard Bossuat (eds.), L'Union européenne, acteur de la sécurité mondiale, Paris : Soleb, 2007, p. 61.

5 Télégramme n̊8690/99, de Seydoux, 22 décembre 1972. AMAE, Europe 1971-76, Organismes internationaux et grandes questions internationales, vol. 2925. 
comme une manifestation de bonne volonté la réévaluation de l'attitude soviétique à l'égard de la construction européenne ; mais on comprend également qu'elle a pour but de faire un geste en direction de l'opposition conservatrice ouest-allemande afin que le Bundestag ratifie au plus vite le traité de Moscou du 12 août 1970.

Pour ne pas perdre la face, le Kremlin justifie l'évolution de sa position en s'appuyant sur une argumentation selon laquelle l'avenir économique de l'Europe ne peut se concevoir sans la participation de l'URSS qui, seule, dispose de ressources naturelles abondantes ${ }^{6}$. Il lie cependant la pleine reconnaissance de la CEE par l'URSS à la reconnaissance du statu quo territorial européen par les Occidentaux.

En parallèle, la diplomatie soviétique s'efforce de rehausser le prestige du Comecon et de le faire admettre par la communauté internationale comme l'homologue de la CEE. C'est dans le cadre de cette nouvelle tactique qu'il faut placer la proposition soviétique d'instaurer des rapports entre les deux instances. Moscou veut éviter que ses satellites noue des relations bilatérales avec la CEE, d'où l'utilisation du Comecon qui en deviendrait l'interlocuteur obligé ${ }^{7}$.

En effet, les autres pays de l'Est adoptent une attitude plus réaliste et, dans certains cas, reconnaissent de facto la Communauté européenne : la Pologne établit des contacts visant à un accord sur les textiles; la Bulgarie, la Hongrie, la Pologne et la Roumanie passent des accords techniques avec la CEE sur le commerce des produits agricoles ; la Roumanie se rapproche du Marché commun pour obtenir un statut de privilégié auprès de la Communauté ${ }^{8}$. Ces pays satellites, dont la majeure partie des produits d'importation provient des Etats ouest-européens, poussent Moscou à trouver rapidement un moyen d'institutionnaliser les relations avec la CEE sans porter atteinte à l'organisation économique du pacte de Varsovie, d'autant que la plupart de leurs accords temporaires passés avec les Neuf arrivent à expiration en 1974.

Malgré cela, l'attitude soviétique à l'égard du Marché commun demeure très méfiante et, encore en 1974, les organes du PCUS parlent de la CEE avec dédain". Celle-ci est toujours perçue comme un ensemble économique fermé et l'embryon d'un nouveau bloc politique, voire militaire ${ }^{10}$.

6 Note de la sous-direction d'Europe orientale, 23 janvier 1974. AMAE, Europe 1971-76, Organismes internationaux..., vol. 2927.

7 Ibidem.

8 Angela Romano, "Western Europe's self assertion towards the superpowers : the CSCE chance and its aftermath", in Anne Deighton, Gérard Bossuat (eds.), L'Union européenne, acteur de la sécurité mondiale, op. cit., p. 158.

9 Note de l'ambassade de France en URSS, 28 janvier 1974. AMAE, Europe 1971-76, URSS, vol. 3721 .

10 Note de la sous-direction d'Europe orientale, 5 mars 1975. AMAE, Europe 1971-76, URSS, vol. 3727 . 
La question de la participation du Comecon à la CSCE est clairement soulevée à l'été 1973. Elle intervient en réaction au débat des Neuf sur les moyens d'intégrer la Commission européenne aux négociations de la conférence. En effet, lors de la première phase de la CSCE, le ministre des Affaires étrangères du Danemark, qui préside la Communauté européenne, annonce l'implication de la Commission dans les futurs travaux de la conférence, dans la mesure où la deuxième corbeille inclut des sujets qui sont de la compétence de la $\mathrm{CE}^{11}$. François-Xavier Ortoli, alors président de la Commission, propose même qu'un représentant de cette dernière soit présent lors des négociations à trente-cinq consacrées à l'exécution du mandat sur les échanges commerciaux et industriels, et qu'il prenne la parole au nom de la Communauté ${ }^{12}$. Tandis que la RFA se montre favorable à ce projet, la France et l'Italie s'y opposent ${ }^{13}$, jugeant que la coordination entre les Neuf pour ce qui est de la CSCE s'effectue dans le cadre de la seule coopération politique et que la conférence ne doit rassembler que des Etats ${ }^{14}$.

Malgré les oppositions, les neuf ministres des Affaires étrangères acceptent, en septembre 1973, de permettre à la Commission d'être représentée au sein de la délégation du pays qui exerce la présidence de la Communauté ${ }^{15}$. Pourtant, à Genève, la France poursuit son action contre une institution qu'elle accuse de conférer trop d'importance à la deuxième corbeille par rapport aux deux autres $^{16}$.

Ainsi, lorsque, constatant l'inclusion progressive de la coopération industrielle dans les compétences de la Commission, le vice-ministre tchécoslovaque des Affaires étrangères insiste auprès du directeur de la section Europe du Quai d'Orsay, Claude Arnaud, sur l'intérêt d'une prise de contact rapprochée entre la CEE et le Comecon, le diplomate français affirme que son pays entend préserver le caractère bilatéral de sa coopération industrielle avec les Etats d'Europe orientale ${ }^{17}$. Il y va de l'image de la France, qui ne souhaite pas que les bénéfices issus de ses rapports anciens et privilégiés avec Moscou lui échappent et profitent à la Commission. Arnaud précise en outre ne pas voir sur quelle base deux organismes

11 Déclaration de la présidence de la Communauté européenne à la première phase de la CSCE, 3 juillet 1973, Helsinki. AMAE, CSCE, vol. 20.

12 Télégramme au départ, 13 août 1973. Archives nationales de France (AN), 5 AG 21041. CSCE. 1969-1974.

13 Note CSCE n³46, 9 octobre 1973. AMAE, Europe 1971-76, RFA, vol. 2982.

14 Télégramme au départ, 13 août 1973. AN, 5 AG 2 1041. CSCE. 1969-1974.

15 Ainsi, ces représentants siègent tour à tour au sein des délégations danoise, allemande, française, irlandaise et italienne. Surtout, il revient à Aldo Moro de parapher l'Acte final d'Helsinki en tant que président du Conseil italien et « en sa capacité de président en exercice du Conseil des Communautés européennes »

16 Document français de 1975, sans date précise. AMAE, CSCE, vol. 20.

17 Entretiens Arnaud/Ruzek, 25 et 26 octobre 1973, Prague. Circulaire n670, 2 novembre 1973. AMAE, CSCE, vol. 33. 
à vocations très différentes pourraient coopérer, évoquant indirectement l'un des problèmes majeurs que pose la deuxième corbeille.

Alors qu'on aurait pu s'attendre à une attitude positive des Français sur la possibilité de développer les relations entre les deux organes, leurs réticences peuvent sembler déroutantes. Elles n'ont pourtant rien d'étonnant : on est conscient à Paris qu'un rapprochement entre la CEE et le Comecon suppose une reconnaissance de la première par les pays qui composent le second. Or, aux yeux du président Georges Pompidou, une telle reconnaissance ne constitue pas un objectif en soi ${ }^{18}$, d'autant plus que Brejnev lie cette dernière à la confirmation, via la CSCE, du statu quo politique et territorial en Europe. La France considère la CSCE comme un moyen de rapprocher les deux moitiés du continent et non comme un instrument destiné à en renforcer la division. L'essentiel, pour le président français, est que les pays de l'Est consentent à s'engager dans le processus d'échanges économiques engagé en Occident depuis le Plan Marshall.

L'autre raison qui explique la réticence de Paris à un rapprochement entre la CEE et le Comecon repose sur leur volonté de ne pas cautionner une nouvelle relation de bloc à bloc qui pourrait gêner certains pays socialistes. Car, outre celle de la France, les voix qui s'élèvent de la manière la plus virulente à l'encontre d'une participation de représentants de la Commission aux travaux de Genève proviennent de l'Est. La Roumanie accuse les Neuf d'introduire la notion de blocs dans une conférence qui vise à surmonter les divisions en Europe ${ }^{19}$. Elle craint surtout, et elle est suivie en cela par la Yougoslavie, que cela donne le droit au Comecon d'en faire de même ${ }^{20}$, ce qui reviendrait - c'est en l'occurrence ce qui arrive par la suite - à faire des négociations de la deuxième corbeille un face-àface entre Moscou et les Neuf. Ce n'est qu'au début du mois de novembre 1973 que les pays socialistes acceptent de voir les représentants de la Commission siéger dans la délégation de la présidence danoise et intervenir dans les domaines qui relèvent des compétences communautaires ${ }^{21}$.

Un tel changement d'attitude n'est pas dû au hasard : la plupart des traités commerciaux Est-Ouest expirent en 1973 et les pays du Comecon, à commencer par l'URSS, prennent conscience qu'avec la caducité, au $1^{\text {er }}$ janvier 1975, des derniers accords bilatéraux qui ont été signés entre eux et les Etats de la CEE, ils vont devoir négocier directement avec la Communauté : le Marché commun constitue un débouché non négligeable pour beaucoup de produits, notamment agricoles, d'Europe de l'Est. Désormais, les pays importateurs de ces produits

18 Entretien Pompidou/Brandt, 11 février 1972, Paris. AN, 5 AG 2 106. RFA. 1972-73.

19 Télégramme $n^{\circ} 1539 / 41$, de Fernand-Laurent, 4 octobre 1973. AMAE, Europe 1971-76, Roumanie, vol. 3537.

20 Note CSCE n`355, 11 octobre 1973. AMAE, Europe 1971-76, Roumanie, vol. 3537.

21 Note pour le président de la République, 8 novembre 1973. AN, 5 AG 2 1015. GrandeBretagne. 1973. 
seront soumis aux quotas imposés par Bruxelles. Il s'agit par conséquent pour les Etats du Comecon de combattre ces pratiques commerciales restrictives en réclamant l'octroi de la clause de la nation la plus favorisée. Or, pour cela, il faut accepter la présence de représentants de la Commission à la CSCE. De fait, grâce à la conférence, les pays du bloc de l'Est se retrouvent pour la première fois impliqués dans des négociations économiques et commerciales directes avec la CEE.

Cependant, les négociations de la deuxième corbeille ne débouchent pas sur une coopération fructueuse entre la Communauté et le Comecon. Les discussions de Genève ne sont d'ailleurs pas celles d'une conférence économique à proprement parler. Contrairement à ce qui se passe dans d'autres instances, on ne négocie pas à la CSCE des mesures précises et détaillées basées sur des tableaux statistiques. On ne cherche pas non plus à atteindre des objectifs chiffrés en termes de volume d'échanges ou de production. La marge de manœuvre des négociateurs est en effet très limitée : la diversité des régimes économiques représentés à Genève et la nature très différente de la $\mathrm{CEE}$ et du Comecon font qu'il est impossible pour les pays de la CSCE de pouvoir engager des discussions concrètes. Le Comecon n'a rien d'un marché commun et constitue essentiellement une instance chargée de coordonner les plans de production nationaux. Les échanges commerciaux et la coopération technique et scientifique en son sein ne s'accomplissent que sur la base d'accords bilatéraux entre les différents pays membres. L'organisation n'émet que des recommandations que ses membres ne sont pas forcés de suivre. Il n'y a donc aucune politique d'échanges extérieurs commune comme dans la $\mathrm{CEE}^{22}$. De fait, 1'objectif soviétique visant à l'amélioration des relations entre la Communauté et le Comecon via la CSCE n'est pas atteint.

Constatant son échec, Moscou s'appuie, à la CSCE, sur une autre de ses revendications pour tirer profit du dynamisme économique des pays de l'Ouest.

\section{Le débat sur la clause de la nation la plus favorisée}

Dès la première phase de la CSCE en juillet 1973, la Hongrie et la RDA déposent un projet de déclaration sur le " développement de la coopération économique, commerciale, scientifique et technique, ainsi que dans le domaine de l'environnement ${ }^{23}$. Ce document met l'accent sur l'élimination des obstacles au développement du commerce. A ce titre, l'objectif majeur de l'URSS, dicté par son besoin de réaliser les premiers plans privilégiant les industries légères et la production de biens de consommation, est d'obtenir l'octroi de la clause de la

22 Note de la sous-direction d'Europe orientale, 14 février 1972. AMAE, Europe 1971-76, RFA, vol. 3017.

23 Note CSCE n²17, 10 juillet 1973. AMAE, Europe 1971-76, Hongrie, vol. 3337. 
nation la plus favorisée. Pour Moscou, la satisfaction de cette requête marquerait la fin de la guerre froide économique et permettrait de contourner les quotas du Marché commun ${ }^{24}$.

L'un des Etats occidentaux les moins gênés par cette demande soviétique est la France, dans la mesure où la clause de la nation la plus favorisée existe déjà dans les accords qu'elle a passés avec les pays de l'Est et que la CEE en applique le régime de jure ou de facto, sans y déceler de danger particulier ${ }^{25}$. Cette application ne touche cependant que le domaine tarifaire et exclut celui des contingents ${ }^{26}$. Or les Soviétiques cherchent justement à obtenir l'extension maximale de la clause, qui engloberait ainsi les contingents et les autres restrictions quantitatives à l'importation ${ }^{27}$.

Quoi qu'il en soit, afin que l'amélioration des échanges commerciaux ne soit pas à sens unique, les Neuf réclament le principe de la réciprocité des échanges ${ }^{28}$, même s'ils sont conscients que seuls des pays, ou dans le cas de la CEE des groupements de pays, à économie de marché peuvent accorder la clause de la nation la plus favorisée. Ainsi, les Occidentaux ne voient pas comment ils peuvent être traités favorablement « par une économie d'Etat qui n'a pas de tarifs et effectue toutes ses importations au moyen de décisions administratives $»^{29}$. Toujours est-il que les Soviétiques demeurent campés sur leur position et refusent toute réciprocité, préférant parler d' " égalité » des droits ${ }^{30}$. Mais les Neuf insistent pour préserver le terme de « réciprocité », qui doit figurer dans le préambule de la deuxième corbeille et être clairement défini comme impliquant que les avantages et obligations pour les parties doivent être d'égale portée ${ }^{31}$. La notion d'« égalité des droits » est inacceptable pour la Commission européenne en ce qu'elle implique la non-discrimination et la libéralisation totale des rapports commerciaux entre les pays socialistes et la Communauté.

Seule la France, parmi les Neuf, se prononce en faveur d'une mention, dans le texte de l'Acte final, de l'égalité des droits demandée par l'Est. Aux yeux des

24 Document du sous-comité CSCE, projet de rapport, 15 mai 1975. AMAE, CSCE, vol. 20.

25 Dépêche $\mathrm{n}^{\circ} 12$ de la direction des Affaires économiques et financières du Quai d'Orsay, 18 juillet 1973. AMAE, CSCE, vol. 26.

26 Les contingents tarifaires subordonnent à une limite fixe la quantité de marchandises pouvant être importée au bénéfice d'une suspension de droits.

27 Dépêche n¹355, de Vimont, 2 juillet 1973. AMAE, CSCE, vol. 26.

28 Note CSCE n¹96, 6 novembre 1974. AN, 5 AG 3 1089. URSS. 1974.

29 Simone Courteix, "La coopération dans les domaines de l'économie, de la science, de la technique et de l'environnement", Annuaire de l'URSS et des Pays socialistes européens 1975, Paris : Librairie Istra, 1977, p. 644.

30 Télégramme n²80/92, de Vimont, 17 janvier 1975. AMAE, Europe 1971-76, URSS, vol. 3689.

31 CPE RM(75)7P, Rapport du président du sous-comité CSCE, 23 mai 1975. AMAE, CSCE, vol. 18. 
Français, parler de l'égalité des partenaires préserverait les chances d'une éventuelle négociation entre la CEE et les pays socialistes ${ }^{32}$. De fait, la délégation française à Genève élabore en février 1975 un texte de compromis, que l'URSS accepte, dans lequel elle reprend à la fois toutes les notions souhaitées par les Neuf et la mention de l'" égalité des droits $»^{33}$.

Pour ce qui est du traitement de la nation la plus favorisée, les Neuf veillent à ce que la formule qui sera retenue ne risque pas d'être interprétée comme constituant en elle-même l'octroi du traitement ou bien comme impliquant que le champ d'application du traitement puisse s'étendre au-delà du domaine tarifaire, à savoir dans le domaine des contingents ${ }^{34}$.

Ce n'est que le 17 juillet 1975 que les experts de la deuxième corbeille parviennent à adopter un projet final englobant les questions essentielles de la clause de la nation la plus favorisée et de la réciprocité des avantages économiques. La formule adoptée évite toute obligation réelle d'accorder la fameuse clause et parvient à compenser l'impossibilité pour une économie socialiste de l'octroyer ${ }^{35}$. Dans le bilan qu'ils font des discussions de la deuxième corbeille, les Neuf considèrent comme « un important progrès pour les relations futures » la reconnaissance de la notion de réciprocité effective dans les échanges commerciaux ${ }^{36}$.

Il ressort des négociations de la deuxième corbeille que les efforts déployés par les diplomates soviétiques dans le but d'utiliser la CSCE pour à la fois développer les rapports commerciaux avec l'Ouest et renforcer l'intégration économique de l'Est ne sont guère fructueux. Au contraire, cette partie de la négociation révèle la cohésion des pays de la Communauté et les faiblesses des échanges EstOuest. L'autre projet soviétique d'intégration, qui a, lui, un caractère paneuropéen, conféré au processus d'Helsinki, n'a pas plus de succès.

\section{Le paneuropéanisme en question : le débat sur l'institutionnalisation de la CSCE}

Depuis 1970, Moscou souhaite la création par la CSCE d'un organe permanent paneuropéen dont la fonction principale serait de prolonger le processus d'Helsinki

32 Document français de 1975, sans date précise. AMAE, CSCE, vol. 20.

33 Télégramme n615/20, de la part de Gérard André, 24 février 1975. AMAE, Europe 197176, URSS, vol. 3689.

34 Document CPE RM(75)2P, 10 février 1975. AMAE, CSCE, vol. 19.

35 Les Etats participants reconnaissent «qu'une telle coopération, en tenant compte des différents niveaux de développement économique, peut-être développée, sur la base d'égalité et de satisfaction mutuelle des partenaires, et de réciprocité, permettant, dans l'ensemble, une répartition équitable des avantages et des obligations d'ampleur comparable, dans le respect des accords bilatéraux et multilatéraux $»$.

36 Document CPE, CP (75) 23 P, Rapport du Comité politique, 8 juillet 1975. AMAE, Europe 1971-76, CEE, vol. 3820. 
et d'y aborder la question du désarmement. Les Polonais vont plus loin en présentant cet organe comme la pièce maîtresse de la CSCE, chargée d'appliquer les décisions de la première conférence et d'en préparer une seconde ${ }^{37}$.

L'ambassadeur de France en URSS, Roger Seydoux, est l'un des premiers à en appeler à la méfiance. Selon lui, avec la création d'un organe permanent paneuropéen dans lequel ne siégeraient pas les Etats-Unis, "l'influence prépondérante de l'URSS en Europe serait en quelque sorte officiellement reconnue, et le désengagement américain confirmé $\aleph^{38}$. Un tel système permettrait à Moscou d'affirmer, à terme, son droit à une présence active en Europe occidentale. En régionalisant l'Europe, il empêcherait le développement de la Communauté européenne, tarirait les possibilités d'élargir les relations avec l'Est et réduirait l'Europe à un rôle marginal puisqu'elle ne pourrait s'appuyer que sur la garantie des superpuissances dont l'une seulement, l'URSS, serait directement insérée dans ledit système en raison de la contiguïté géographique ${ }^{39}$.

Très vite, les neuf membres de la Communauté se montrent unanimes. Un élément essentiel vient cependant les gêner, les Français surtout : au cours de l'année 1972, la Roumanie prend la tête des pays désireux de créer un organe au sein duquel tous les Etats européens siégeraient sur un pied d'égalité, en dehors des blocs, et qui veillerait au respect des principes d'Helsinki, en particulier celui de l'indépendance des Etats ${ }^{40}$. Comment la France, dont la politique européenne demeure largement fondée sur les idées gaulliennes de souveraineté, d'égalité et d'indépendance des Etats, peut-elle repousser un pareil projet sans perdre sa crédibilité dans les pays d'Europe de l'Est soumis au joug soviétique ? Cette question prend un caractère central au sein de l'appareil diplomatique français au cours des mois de préparation de la CSCE. On peut y voir un joli coup tactique de la part du Kremlin : bien que développant une politique destinée à démontrer qu'elle dispose d'une relative marge de manœuvre par rapport à l'URSS, la Roumanie est toujours, en 1972, un satellite de Moscou. Encerclée par des pays communistes, loin de l'Allemagne et du rideau de fer, disposant de l'un des régimes les plus durs du pacte de Varsovie, la Roumanie ne constitue pas vraiment un danger aux yeux des Soviétiques. Au contraire, ces derniers profitent des velléités roumaines pour laisser paraître au reste du monde que leur mainmise sur l'Europe orientale n'est pas aussi redoutable qu'on veut bien le croire.

37 Circulaire n³49, de Beaumarchais, 4 octobre 1971. AMAE, Europe 1971-76, Pologne, vol. 3476 .

38 Télégramme $n^{\circ} 7592 / 7600$, de Seydoux, 3 novembre 1972. AMAE, Europe 1971-76, Organismes..., vol. 2925.

39 Dépêche non signée et non datée précisément, 1972. AMAE, CSCE, vol. 1.

40 Note de la sous-direction d'Europe orientale, 14 avril 1972. AMAE, Europe 1971-76, Roumanie, vol. 3537. 
Toujours est-il que les Français comprennent que des pays comme la Roumanie, la Yougoslavie, voire l'Autriche ou la Finlande souhaitent disposer, après la CSCE, d'une instance de recours pour les cas où ils se sentiraient menacés ${ }^{41}$. On considère donc que l'unique condition pouvant permettre la mise sur pied d'un tel organe est que la CSCE produise des résultats positifs dans l'ensemble des domaines qu'elle aborde ; il s'agit de ne pas donner l'impression que la conférence a déjà réussi à transformer les relations entre Etats ${ }^{42}$. La France conçoit une institutionnalisation de la CSCE seulement si l'URSS et ses alliés se décident à davantage de concessions sur le changement pacifique des frontières, les droits de l'homme et la coopération culturelle et s'ils appliquent par la suite les dispositions de l'Acte final.

C'est finalement une proposition émanant du Danemark qui l'emporte : elle prévoit une période intérimaire de quelques années durant lesquelles les Etats participants s'attacheraient à mettre leurs engagements en œuvre, suivie d'une réunion de représentants officiels en 1977. L'institutionnalisation des suites ne serait possible qu'à l'issue de la période probatoire, selon le degré de respect des dispositions de l'Acte final. L'idée danoise permet ainsi aux Neuf de garder un maximum de contrôle sur l'évolution ultérieure du processus multilatéral ${ }^{43}$. Elle leur permet également de combattre l'idée soviétique selon laquelle la CSCE représente un aboutissement, la consécration définitive du statu quo politique et territorial en Europe.

La tendance des Neuf, appuyés par les $\mathrm{Neutres}^{44}$, consistant à lier la question des suites aux résultats de la CSCE produit peu à peu ses effets : après une dernière déclaration sur le sujet le 19 avril 1974, les Soviétiques et leurs alliés se montrent soudainement moins intéressés par ce problème. Ils prennent conscience du danger que pourrait créer la filiation entre suites institutionnelles et application des dispositions de la conférence, particulièrement en matière d'échanges culturels et de contacts humains.

Le recul de l'URSS et la prudence des Neuf rendent finalement unanime la décision d'adopter la proposition danoise sur les suites. En accord avec le document ouest-européen, on assigne à la réunion de hauts fonctionnaires prévue en 1977, précédée d'une réunion préparatoire, la tâche d'évaluer la mise en œuvre des décisions de la CSCE et l'état des relations entre les Etats participants ainsi que de présenter des propositions concernant des mesures propres à permettre d'atteindre

41 Entretien Pompidou/Brandt, 22 janvier 1973, Paris. AN, 5 AG 2 106. RFA. 1972-73.

42 Note CSCE, 5 janvier 1974. AMAE, Europe 1971-76, URSS, vol. 3689.

43 Document CPE, rapport du président du sous-comité CSCE, 23 mai 1975. AMAE, CSCE, vol. 18.

44 Chez les Neutres et Non-alignés, seules la Yougoslavie et l'Espagne se prononcent clairement en faveur d'un organisme permanent. Les Espagnols exercent une pression sur les Français pour les faire changer d'avis, tentant, de la sorte, de compenser leur déception d'être tenus à l'écart de la CEE et de l'Alliance atlantique. Entretien de Courcel/Gehlhoff, 21 juillet 1975, Bonn. AMAE, Europe 1971-76, RFA, vol. 2999. 
les objectifs de la conférence ${ }^{45}$. En juillet 1975, seule la Roumanie, par la voix de Ceausescu lui-même, en appelle encore, en vain, à la création d'un organisme permanent ${ }^{46}$. En fin de compte, quand intervient la signature de l'Acte final d'Helsinki le $1^{\text {er }}$ août 1975 , l'URSS n'est parvenue à mener à son terme aucun des deux objectifs qu'elle s'était fixés en matière d'intégration européenne : elle n'a pas réussi à renforcer suffisamment la cohésion du Comecon pour en faire l'équivalent de la CEE ; elle n'a pu convertir les Occidentaux au paneuropéanisme. Ni son projet d'intégration est-européenne ni son dessein d'intégration paneuropéenne n'ont été réalisés lors de la CSCE. Sur l'un comme sur l'autre, la conférence a abouti au résultat inverse : elle a renforcé la propension des pays du bloc communiste à négocier bilatéralement avec la $\mathrm{CE}$ et la Commission européenne, discréditant un peu plus le Comecon; elle a renforcé la méfiance des pays de l'Ouest à l'égard de tout ce qui relève de la sécurité collective et a mis en exergue le rôle dynamique des Neuf.

La deuxième corbeille a également le mérite d'essayer de poser les bases multilatérales de la détente économique et, par certains aspects - comme le développement des échanges commerciaux ou l'étude et la réalisation de projets industriels et de transports importants - peut aider les pays du Comecon à accéder à la technologie occidentale tout en permettant aux Etats capitalistes de conclure des contrats à long terme avec l'Est. Pour les Neuf, qui se félicitent que le rôle des entreprises et, le cas échéant, celui des individus soient reconnus, les textes de la deuxième corbeille constituent « une contribution positive au développement de la coopération $»^{47}$. Si la CSCE ne renforce ni l'intégration est-européenne ni l'intégration paneuropéenne, au moins est-elle le fruit d'un vrai compromis entre les deux visions de l'économie qui prévalent de part et d'autre du rideau de fer.

\section{Bibliographié}

Courteix, Simone, (1977), "La coopération dans les domaines de l'économie, de la science, de la technique et de l'environnement", Annuaire de l'URSS et des Pays socialistes européens 1975, Paris: Librairie Istra.

Du Réau, Elisabeth et Frank, Robert (eds.), (2002), Dynamiques européennes : nouvel espace, nouveaux acteurs, 1969-1981, Paris: Publications de la Sorbonne.

Ludlow, N. Piers (ed.), (2007), European integration and the Cold War: Ostpolitik-Westpolitik, 1965-1973, London: Routledge.

45 Projet de rapport du sous-comité CSCE, 15 mai 1975. AMAE, CSCE, vol.20.

46 Début juillet 1975, Ceausescu demande, en vain, à Sauvagnargues de le soutenir dans sa demande. Il réitère son désir d'un organe permanent dans son discours à Helsinki. Télégramme au départ n562/65, de Laboulaye, 9 juillet 1975. AMAE, Europe 1971-76, Roumanie, vol. 3537.

47 Document CPE, CP (75) 23 P, Rapport du Comité politique, 8 juillet 1975. AMAE, Europe 1971-76, CEE, vol. 3820. 
Möckli, Daniel, (2008), European Foreign Policy during the Cold War: Heath, Brandt, Pompidou and the Dream of Political Unity, 1969-1974, Londres: I.B Tauris.

Rey, Marie-Pierre, (2007), "L'URSS et l'Europe communautaire, représentations et pratiques, 1957-1991”, in Deighton, Anne et Bossuat, Gérard (eds.), L’Union européenne, acteur de la sécurité mondiale, Paris : Soleb, pp. 48-67.

Rey, Marie-Pierre, (2005), "Le retour à l'Europe? Les décideurs soviétiques face à l'intégration ouest-européenne, 1957 - 1991”, in Journal of European Integration 1, 11, pp. 7-27.

Romano, Angela, (2009), From Détente in Europe to European Détente. How the West shaped the Helsinki CSCE, Bruxelles: PIE-Peter Lang.

Romano, Angela, (2007), "Western Europe's self assertion towards the superpowers : the CSCE chance and its aftermath", in Deighton, Anne et Bossuat, Gérard (eds.), L'Union européenne, acteur de la sécurité mondiale, Paris : Soleb, pp. 148-165.

Takeshi, Yamamoto, (2007), "Détente or Integration? EC Response to Soviet Policy Change towards the Common Market, 1070-75”, in Cold War History, Vol. 7, N¹, pp. 75-94.

Wenger, Andreas, Mastny, Vojtech et Nuenlist, Christian (eds.), (2008), Origins of the European Security System: the Helsinki Process revisited. 1965-1975, London: Routledge. 